\title{
Die Überprüfung des EU-Haushalts 2009: Reform- perspektiven für eine zukunftsorientierte Ausgabenstruktur
}

\author{
Robert Kaiser und Heiko Prange-Gstöhl*
}

Die Überprüfung des Haushalts der Europäischen Union zur Halbzeit eines Mehrjährigen Finanzrahmens ist ein Novum in der Integrationsgeschichte. Zum ersten Mal überhaupt hat der Europäische Rat die Kommission eingeladen, ${ }^{1}$ eine vollständige und umfassende Überprüfung der Ausgaben der Union zur Halbzeit des Mehrjährigen Finanzrahmens 2007 bis 2013 vorzunehmen. Diese Überprüfung betrifft sensible Themen der EU-Finanzierung, seien es die Ausgaben für die Gemeinsame Agrarpolitik oder die britischen Ausgleichszahlungen.

Die Kommission unter José Manuel Barroso hat diese Initiative aufgenommen und im Jahr 2007 angekündigt, die Überprüfung unvoreingenommen und , ohne Tabus ‘ durchzuführen. Aus ihrer Sicht stellt dieser Prozess eine einzigartige Möglichkeit dar, die zukünftigen Finanzierungsprioritäten der Union zu beeinflussen, ohne dabei unter dem unmittelbaren Entscheidungsdruck von Verhandlungen über den kommenden Mehrjährigen Finanzrahmen (ab 2014) zu stehen. ${ }^{2}$

Ohne Zweifel besteht Anlass, die derzeitige Ausgabenstruktur der Europäischen Union kritisch zu reflektieren. Der eher schleichende Fortschritt der Lissabon-Agenda für Wettbewerbsfähigkeit, Wachstum und Beschäftigung ist ein Grund hierfür, zumal die Europäische Union in diese Initiative bisher nur relativ geringe Mittel investiert. So machen die Ausgaben für die sogenannten Lissabon-Politiken derzeit weniger als 10 Prozent des Haushalts aus. Dabei ist auch zu berücksichtigen, dass die Mitgliedstaaten bei der Verabschiedung des aktuellen Mehrjährigen Finanzrahmens den diesbezüglichen Etatvorschlag der Europäischen Kommission um immerhin 46 Prozent gekürzt hatten. Damit behielt der EU-Haushalt im Kern seinen anachronistischen Bias zugunsten von Landwirtschafts- und Regionalpolitik, wobei insbesondere Letztgenannte zu häufig dazu genutzt wird, veraltete Industrien zu stützen. ${ }^{3}$

Vor diesem Hintergrund wird davon ausgegangen, dass die Überprüfung des EU-Haushalts signifikante Auswirkungen auf verschiedene Gemeinschaftspolitiken haben könnte und zwar insoweit, als dieser Prozess eine determinierende Wirkung auf die erst im Jahr 2011 beginnenden Verhandlungen über den nächsten Finanzrahmen entfalten kann. Diese Annahme begründet sich zunächst aus zwei Entscheidungen der Europäischen Kommission hinsichtlich des Ablaufs der Haushaltsüberprüfung.

\footnotetext{
* PD Dr. Robert Kaiser, Lehrstuhl für Vergleichende Politikwissenschaft, Ludwig-Maximilians-Universität München.

Dr. Heiko Prange-Gstöhl, Direktion Internationale Kooperation, Generaldirektion Forschung der Europäischen Kommission, Brüssel.

Der Beitrag spiegelt die persönlichen Ansichten der Autoren wider und stellt in keiner Weise die offizielle Position der Europäischen Kommission dar.

1 Rat der Europäischen Union: Finanzielle Vorausschau 2007-2013, Dok. 15915/05.

2 Europäische Kommission: Mitteilung der Kommission. Den Haushalt reformieren, Europa verändern. Konsultationspapier im Hinblick auf die Überprüfung des EU-Haushaltes, SEK (2007) 1188.

3 Ian Begg/Friedrich Heinemann: New Budget, Old Dilemmas, Centre for European Reform: CER Briefing Note, Februar 2006, S. 3.
} 
$\mathrm{Zu}$ diesen Entscheidungen gehörte, erstens, dass alle EU-Politikbereiche mit größeren Ausgabenprogrammen, also insbesondere die Gemeinsame Agrarpolitik, die Regional- und Kohäsionsförderung sowie die Forschungs- und Technologiepolitik, einen ,GesundheitsCheck' durchlaufen werden, wobei die Halbzeitbilanz des derzeitigen ,Siebten Rahmenprogramms für Forschung, technologische Entwicklung und Demonstration" (Siebtes Forschungsrahmenprogramm) erst im Jahr 2010 ansteht. Eine zweite Entscheidung der Kommission bestand darin, zur Überprüfung des Haushalts einen umfassenden Prozess der öffentlichen Anhörung zu initiieren. In diesem Zusammenhang haben sich die Regierungen der Mitgliedstaaten verpflichtet, eigene Stellungnahmen abzugeben und dabei ihre Prioritäten und Interessen zu benennen. Weitere politische Interessensbekundungen waren zudem vom Europäischen Parlament und den nationalen Parlamenten zu erwarten, die ebenfalls durch den Rat aufgefordert wurden, ihre Positionen zu übermitteln.

Sofern die Überprüfung des Haushalts also tatsächlich Vorfestlegungen zugunsten einschneidender Veränderungen in Bezug auf die zukünftige Finanzierung neuer EU-Aufgabenprioritäten begründen wird, so dürfte sich dies im Ergebnis in unterschiedlichen Graden möglichen Politikwandels konzeptionalisieren lassen. Hier folgen wir der einschlägigen Differenzierung von Peter Hall über Wandel erster, zweiter und dritter Ordnung. ${ }^{4}$

Politikwandel erster Ordnung würde in diesem Kontext lediglich erwarten lassen, dass es zu einer begrenzten Anpassung bestehender Instrumente und Programme innerhalb der jeweiligen Politikbereiche kommt. Ein solches Szenario wäre insbesondere dann denkbar, wenn weder eine signifikante Zahl mitgliedstaatlicher Regierungen noch zivilgesellschaftliche Akteure einen erheblichen politischen Druck zugunsten einer neuen Haushaltsstruktur ausüben und insofern wenig Anlass für die Europäische Kommission bestehen würde, Unterstützung für weitreichende Veränderungsvorschläge zu sehen.

Politikwandel zweiter Ordnung würde hingegen eine Situation beschreiben, in der sowohl die Herausforderungen der Lissabon-Agenda wie auch die globale Wirtschafts- und Finanzkrise entweder innerhalb existenter Programmstrukturen eine Erhöhung der Ressourcen begründen würde und/oder es zu einer signifikanten Verschiebung von Ressourcen innerhalb der Politikbereiche zugunsten der Lissabon-Ziele kommt. Eine solche Situation erscheint aber nur im Falle eines weitgehenden Konsenses zwischen Rat, Parlament und Kommission denkbar, der zudem durch wichtige gesellschaftliche Akteure unterstützt würde.

Politikwandel dritter Ordnung oder die Etablierung eines neuen Paradigmas europäischer Politikgestaltung erscheint demgegenüber zunächst die geringste Wahrscheinlichkeit zu besitzen. Hier wäre zu erwarten, dass der Gemeinschaftshaushalt grundlegend in Richtung auf die Lissabon-Agenda und die neuen globalen Herausforderungen hin ausgerichtet würde. Dies würde nicht nur eine erhebliche Erhöhung der finanziellen Ressourcen erfordern, sondern gleichzeitig auch eine Fokussierung auf Instrumente und Programme, bei denen die Europäische Union tatsächlich eine kritische Masse erreichen kann. Ein solcher Paradigmenwechsel erscheint aber nur möglich, wenn eine größere Zahl von Mitgliedstaaten das Agenda-Setting bestimmt, weil ein Politikwandel dritter Ordnung auch auf Seiten der Europäischen Kommission unzweifelhaft Gewinner und Verlierer produzieren würde und insofern nicht davon auszugehen wäre, dass eine entsprechend radikale Umorientierung von ihr selbst vorgeschlagen würde.

Im Folgenden wird unsere Analyse zunächst den inneren und äußeren Reformbedarf thematisieren, dem sich die Europäische Union durch die Lissabon-Agenda und die neuen globalen Herausforderungen ausgesetzt sieht. Im nächsten Schritt werden die möglichen Auswirkungen

4 Peter Hall: Policy Paradigms, Social Learning, and the State: The Case of Economic Policy-Making in Britain, in: Comparative Politics 3/1993, S. 275-296. 
der Haushaltsüberprüfung auf jene Politikbereiche, die aufgrund ihrer Auf- und Ausgabenstruktur im Fokus stehen, konkretisiert. Dies sind insbesondere die Gemeinsame Agrar-, die Regional- sowie die Forschungs- und Technologiepolitik. Abschließend benennen wir die strukturellen Elemente und Bedingungen der drei Szenarien, die unserer Auffassung nach als Reformperspektiven für einen zukunftsfähigen Haushalt der Europäischen Union existieren.

\section{Die Überprüfung des EU-Haushalts: Reformbedarf und Reformdruck}

Interner Reformbedarf, hervorgerufen vor allem durch den bisher mäßigen Erfolg der Lissabon-Strategie, sowie externer Reformdruck, insbesondere als Folge neuer globaler Herausforderungen sowie der internationalen Finanz- und Wirtschaftskrise, werden die zentrale Rolle bei der Überprüfung des EU-Haushalts und somit für die hierauf basierenden Vorschläge der Kommission spielen.

\section{Interner Reformbedarf durch die Lissabon-Strategie}

Bereits im Jahr 2004 hat der sogenannte Kok-Report auf den dringenden Bedarf hingewiesen, Forschung und Entwicklung (F\&E) in den Fokus europäischer Reformpolitik zu rücken. ${ }^{5}$ Die Kommission hat daraufhin eine Neuauflage der Lissabon-Strategie in Form einer Europäischen Partnerschaft vorgeschlagen, ${ }^{6}$ welche von den Staats- und Regierungschefs der Europäischen Union im Frühjahr 2005 angenommen wurde. ${ }^{7}$ Diese Neuauflage basiert im Wesentlichen auf drei Pfeilern:

1. „Stärkung der Anziehungskraft Europas für Investoren und Arbeitskräfte“:

- Erweiterung und Vertiefung des Binnenmarkts,

- Verbesserung der europäischen und der nationalen Vorschriften,

- Gewährleistung offener und wettbewerbsorientierter Märkte innerhalb und außerhalb Europas,

- Erweiterung und Verbesserung der europäischen Infrastruktur.

2. „Wissen und Innovation für Wachstum“:

- mehr und gezieltere Investitionen in Forschung und Entwicklung,

- Erleichterung der Innovation, Verbreitung von Informations- und Kommunikationstechnologie und schonende Nutzung der Ressourcen,

- Beitrag zu einer starken industriellen Basis in der Europäischen Union.

3. „Mehr und bessere Arbeitsplätze schaffen“:

- mehr Menschen an das Erwerbsleben heranführen und die Sozialschutzsysteme modernisieren,

- die Anpassungsfähigkeit von Arbeitnehmern und Unternehmen sowie die Flexibilität der Arbeitsmärkte steigern,

- mehr in das Humankapital investieren: bessere Bildung und Qualifikation.

Die Implementierung der Lissabon-Strategie erfordert ein erhebliches Engagement nicht nur der Gemeinschaft, sondern auch der EU-Mitgliedstaaten selbst. Die Europäische Kommission hat seit dem Frühjahr 2005 verschiedene Initiativen ergriffen, um die Umsetzung der Strategie politisch zu unterstützen. Im Oktober 2005 hat sie beispielsweise 19 Aktions-

5 Bericht der Hochrangigen Sachverständigengruppe unter Vorsitz von Wim Kok: Die Herausforderung annehmen. Die Lissabon-Strategie für Wachstum und Beschäftigung. November 2004, Luxemburg 2004, S. 6.

6 Europäische Kommission: Mitteilung für die Frühjahrstagung des Europäischen Rates. Zusammenarbeit für Wachstum und Arbeitsplätze. Ein Neubeginn für die Strategie von Lissabon, KOM (2005) 24, S. 6-9.

7 Rat der Europäischen Union: Tagung des Europäischen Rates (Brüssel, 22./23. März 2005). Schlussfolgerungen des Vorsitzes, Dok. 7619/1/05. 
felder für die Gemeinschaft und ihre Mitgliedstaaten beschrieben, um die Ziele von ,Lissabon` im Bereich Forschung, Entwicklung und Innovation erreichen zu können. Dazu zählen Initiativen zur Verbesserung der Rechtsetzung für neue Technologien, die Nutzung des öffentlichen Auftragswesens für die Förderung von Forschung und Innovation, die Nutzung der Europäischen Strukturfonds als Motoren für Forschung und Innovation sowie eine Verbesserung des Zugangs von klein- und mittelständischen Unternehmen zu Finanzmitteln. Forschung und Innovation sollen vor allem als ein vorrangiger Bereich der nationalen Reformprogramme für Wachstum und Beschäftigung behandelt werden. ${ }^{8}$

Außerdem hat die Kommission einen Zehn-Punkte-Aktionsplan aufgestellt, der gezielt helfen soll, die Innovationsfähigkeit der Gemeinschaft zu stärken. Das Programm konzentriert sich dabei vor allem auf die Verbesserung des Marktzugangs für neue innovative Produkte und Dienstleistungen auf sogenannten ,Lead'-Märkten. Die Vorschläge - die eine Antwort auf den Bericht der sogenannten Aho-Expertengruppe ${ }^{9}$ sind - reichen von der Aufforderung, einen offenen und freien Arbeitsmarkt für Forscher zu etablieren, über die Ankündigung der Kommission eine neue Patentstrategie vorzulegen, bis hin zur Absicht, die Kohäsionspolitik für innovative Maßnahmen auf regionaler Ebene zu öffnen. ${ }^{10}$

Zur Umsetzung der Lissabon-Agenda schlägt die Kommission sogenannte integrierte Leitlinien vor, die vom Ministerrat genehmigt werden und die Basis für die Nationalen Reformprogramme der Mitgliedstaaten bilden. Gleichzeitig entwickelt die Kommission ein Lissabon-Programm der Gemeinschaft, mit welchem sie sicherstellen will, dass alle Gemeinschaftspolitiken und -finanzierungen bestmöglich dem europäischen Wachstums- und Beschäftigungsziel dienen. All dies geschieht in einem Drei-Jahres-Zyklus. Zwar fällt der Kommission eine Überwachungsfunktion in Bezug auf die Implementierung der Nationalen Reformprogramme zu, die Umsetzung der Programme ist für die Mitgliedstaaten allerdings freiwillig, sodass im Falle der Missachtung keine Sanktionen drohen.

\section{Externer Reformdruck: neue globale Herausforderungen und die internationale Wirt- schafts- und Finanzkrise}

Unterschiedliche Entwicklungen, insbesondere die steigende Nachfrage nach Energie, Nahrungsmitteln und Rohstoffen in dynamischen Ökonomien wie China und Indien sowie der unverminderte Anstieg des klimaschädlichen Kohlendioxidausstoßes, haben europäische Regierungen in den letzten Jahren sensibler auf derartige globale Herausforderungen reagieren lassen. Sie befürchten, dass diese Entwicklungen - in Kombination mit einer wachsenden globalen wirtschaftlichen Kluft zwischen Arm und Reich - die Gefahr politischer Instabilitäten, gewaltsamer Konflikte und des Terrorismus erhöhen werden.

Die Europäische Union sieht sich selbst in der führenden Position bei der Formulierung von internationalen Antworten auf und regulativer Standards für derartige Schlüsselthemen, die an nationalen Grenzen nicht Halt machen. ${ }^{11}$ Ein deutlicher Hinweis auf ein signifikantes

8 Europäische Kommission: Umsetzung des Lissabon-Programms der Gemeinschaft: Mitteilung der Kommission an den Rat, das Europäische Parlament, den Europäischen Wirtschafts- und Sozialausschuss und den Ausschuss der Regionen. Mehr Forschung und Innovation - In Wachstum und Beschäftigung investieren: Eine gemeinsame Strategie, KOM (2005) 488.

9 „Unabhängige Sachverständigengruppe für F\&E und Innovation“.

10 Europäische Kommission: Mitteilung der Kommission an den Europäischen Rat (Informelles Treffen in Lahti (Finnland) am 20. Oktober 2006). Ein innovationsfreundliches, modernes Europa, KOM (2006) 589; Europäische Kommission: Mitteilung der Kommission an den Rat, das Europäische Parlament, den Europäischen Wirtschafts- und Sozialausschuss und den Ausschuss der Regionen. Kenntnisse in die Praxis umsetzen: Eine breit angelegte Innovationsstrategie für die EU, KOM (2006) 502.

11 Europäische Kommission: Mitteilung der Kommission an den Europäischen Rat vom Juni 2006. Europa in der Welt - Praktische Vorschläge für mehr Kohärenz, Effizienz und Sichtbarkeit, KOM (2006) 278. 
Engagement der Europäischen Union in dieser Hinsicht wäre die Höhe der Investitionen in Forschung und Entwicklung für globale Herausforderungen. Allerdings ist die Feststellung derartiger Investitionen äußerst schwierig, da die Budgetlinien nur sehr allgemeine Angaben über die durchgeführten Aktivitäten machen. ${ }^{12}$ So sind die F\&E-Investitionen für generelle umweltbezogene Ziele beispielsweise in verschiedene Maßnahmen integriert und nicht in einem einzigen Budget konzentriert. ${ }^{13}$ Feststeht allerdings, dass das Siebte Forschungsrahmenprogramm 8,5 Milliarden Euro für Umwelt-, Energie- und Transportforschung aufwendet. Dies sind die einzigen Mittel im EU-Haushalt, die zweifelsfrei für den Kampf gegen den Klimawandel identifiziert werden können.

Der Reformdruck durch neue externe Herausforderungen wird in den Stellungnahmen zur EU-Haushaltsüberprüfung deutlich. Industrie, Nicht-Regierungsorganisationen, Wissenschaftler und andere Interessierte waren einhellig der Auffassung, dass die EU-Ausgaben zukünftig auf die Bereiche Forschung, Kampf gegen den Klimawandel, Energiesicherheit und andere Wachstumsbereiche ausgerichtet werden müssen.

Der externe Reformdruck ist durch die internationale Wirtschafts- und Finanzkrise zweifellos noch weiter angestiegen. Im Dezember 2008 hat der Europäische Rat ein Europäisches Konjunkturprogramm verabschiedet, das einem Finanzumfang von circa 1,5 Prozent des EU-Bruttoinlandsprodukts - oder 200 Milliarden Euro - entspricht. ${ }^{14}$ Mit dem Europäischen Konjunkturprogramm gibt die Europäische Union einen Hinweis, in welche Richtung der Haushalt zukünftig ausgerichtet sein könnte. Basierend auf den Vorschlägen der Europäischen Kommission von November $2008^{15}$ beschreibt das Programm sogenannte , smart investments ' in die ,Fähigkeiten der Zukunft', in Energieeffizienz, in , saubere ' Bau- und Automobiltechnologien und in Infrastrukturmaßnahmen. Dieses Programm soll vor allem die Lissabon-Strategie unterstützen, indem die Investitionen in Forschung und Entwicklung insbesondere mithilfe öffentlich-privater Partnerschaften gesteigert werden. Auf der Gemeinschaftsebene besteht das Programm aus drei Komponenten:

Erstens will die Kommission drei solcher öffentlich-privater Partnerschaften etablieren, um Innovationen in der verarbeitenden Industrie, insbesondere im Bereich , grüner ‘ Technologien, zu unterstützen: ${ }^{16}$

- Eine „Europäische Initiative für umweltfreundliche Kraftfahrzeuge“ in einem Umfang von ungefähr fünf Milliarden Euro, davon circa eine Milliarde Euro für Forschung und Entwicklung. Neben der Gemeinschaft und der Industrie sollen auch die nationalen Regierungen sowie die Europäische Investitionsbank zur Finanzierung beitragen.

- Eine „Europäische Initiative für energieeffiziente Gebäude“ in einem Umfang von ungefähr einer Milliarde Euro für Forschung und Entwicklung.

- Eine „Initiative zur Fabrik der Zukunft“, um die Technologienutzung im produzierenden Gewerbe zu fördern. Der Umfang dieser Initiative beträgt ungefähr 1,2 Milliarden Euro für Forschung und Entwicklung.

12 Siehe Europäische Kommission: Results of the Public Consultation on the Green Paper "The European Research Area: New Perspectives", SEC (2008) 430, S. 142.

13 Camilla Adelle/Marc Pallemaerts/David Baldock: Turning the EU Budget into an Instrument to Support the Fight against Climate Change, Swedish Institute for European Policy Studies: SIEPS Report 4/2008, S. 13.

14 Rat der Europäischen Union: Tagung des Europäischen Rates vom 11./12. Dezember 2008 in Brüssel. Schlussfolgerungen des Vorsitzes, Dok. 17271/1/08.

15 Europäische Kommission: Mitteilung der Kommission an den Europäischen Rat. Europäisches Konjunkturprogramm, KOM (2008) 800 .

16 Ebenda, S. 17-18. 
Während diese Initiativen grundsätzlich sicherlich sinnvolle Maßnahmen darstellen, liegt der Teufel im Detail, insbesondere hinsichtlich der Umsetzung. Zunächst muss die Kommission ihren finanziellen Anteil in den jährlichen Arbeitsprogrammen des Siebten Forschungsrahmenprogramms identifizieren. Da der finanzielle Rahmen begrenzt ist, müssen die insgesamt 1,5 Milliarden Euro über die nächsten Jahre sukzessiv aufgebracht werden. Für das Jahr 2010 sind 350 Millionen Euro für die oben genannten Initiativen vorgesehen. Projekte werden über den üblichen Aufruf für die Einreichung von Projektvorschlägen ausgewählt. Dieses Verfahren entspricht jedoch noch nicht den anvisierten langfristigen öffentlich-privaten Partnerschaften.

Zudem muss die Kommission die Industrie motivieren, sich in den geplanten Partnerschaften finanziell zu engagieren. Zu diesem Zweck hatte sich Ende März 2009 EU-Forschungskommissar Janez Potočnik erstmals mit Vertretern Europäischer Technologieplattformen getroffen. Die Industrie hat sich in diesem Gespräch weder in Bezug auf die Höhe noch auf die Form eines eventuellen Engagements festgelegt. ${ }^{17}$ Die in der Gemeinsamen Erklärung erwähnten ,matching investments " könnten auch durch die Beteiligung an kooperativen EUProjekten, das heißt ohne eine formalisierte öffentlich-private Partnerschaft, geleistet werden. Längerfristigere institutionalisierte Partnerschaften, zum Beispiel in Form Gemeinsamer Technologieinitiativen, werden an administrative Reformen geknüpft. ${ }^{18}$ Insgesamt scheint sich die Industrie somit eher zurückzuhalten und zunächst den Start der relevanten EU-Projekte im Jahr 2010 abwarten zu wollen, bevor sie längerfristigere Verpflichtungen eingeht.

Zweitens hat die Kommission angekündigt, dass die Europäische Investitionsbank die sogenannte ,Fazilität für Finanzierungen auf Risikoteilungsbasis ‘ verstärkt einsetzen wird, um die Forschungs- und Entwicklungstätigkeit des privaten Sektors zu stimulieren. ${ }^{19}$ Dieses Instrument soll vor allem die Finanzierung großer Forschungsprojekte und -infrastrukturen garantieren. Das Siebte Forschungsrahmenprogramm unterstützt die Fazilität mit einer Milliarde Euro, um insgesamt circa sechs Milliarden Euro für Forschungsprojekte zu mobilisieren.

Und drittens sieht das Konjunkturprogramm vor, Energiedurchleitungssysteme und Breitbandinfrastrukturen zu fördern. Die Kommission hat zu diesem Zweck im Dezember 2008 vorgeschlagen, den Mehrjährigen Finanzrahmen zu revidieren und so in den Jahren 2009 und 2010 fünf Milliarden Euro aus dem EU-Haushalt zu mobilisieren. ${ }^{20}$

Nach einem langwierigen Verhandlungsprozess zwischen der Kommission, den EU-Mitgliedstaaten und dem Europäischen Parlament konnte Ende April 2009 eine endgültige Einigung über die Finanzierung des Konjunkturprogramms sowie die Änderung des Mehrjährigen Finanzrahmens gefunden werden. Von den insgesamt fünf Milliarden Euro werden

17 Siehe Joint Statement, European Economic Recovery Plan 2010-2013: Public Private Partnerships in Research Activities, in: Europäische Kommission: Europäisches Konjunkturprogramm: Kommission und Industrie vereinbaren zügige Einrichtung öffentlich-privater Partnerschaften für Forschung und Innovation, Pressemitteilung, IP/09/520, 01.04.2009.

18 Im Siebten Forschungsrahmenprogramm wurde ex-Artikel 171 des Vertrags zur Gründung der Europäischen Gemeinschaft (EGV) („Die Gemeinschaft kann gemeinsame Unternehmen gründen oder andere Strukturen schaffen, die für die ordnungsgemäße Durchführung der Programme für gemeinschaftliche Forschung, technologische Entwicklung und Demonstration erforderlich sind.") genutzt, um Gemeinsame Technologieinitiativen (GTI) zu starten. Obwohl die Nutzung von ex-Artikel 171 EGV auch in diesen Fällen konsequent erscheint, bedarf seine Implementierung eines Zeitraums, der einer raschen Reaktion auf die Krise eher widerspricht. Der Grund ist, dass Entscheidungen gemäß ex-Artikel 171 EGV vom Rat mit qualifizierter Mehrheit auf Vorschlag der Kommission und nach Konsultierung des Europäischen Parlaments getroffen werden müssen. Bei bestehenden GTIs ist zudem eine längere Konsultationsphase mit der Industrie vorangegangen, bevor die Kommission einen Vorschlag vorgelegt hat.

19 Europäische Kommission: Europäisches Konjunkturprogramm, 2008, S. 14.

20 Europäische Kommission: Mitteilung der Kommission an das Europäische Parlament und den Rat über die Änderung des mehrjährigen Finanzrahmens (2007-2013), KOM (2008) 859, S. 17. 
lediglich zwei Milliarden Euro durch eine Revision des Mehrjährigen Finanzrahmens finanziert, der Rest über normale Budgetprozeduren (Kompensationsmechanismus) oder Umwidmung innerhalb einer Budgetlinie. ${ }^{21}$

Nach der Übereinkunft, die auf der Basis eines Kompromissvorschlages der tschechischen Präsidentschaft erzielt wurde, sollen 3,98 Milliarden Euro für Energieprojekte (davon 2,365 Milliarden Euro für Gas- und Elektrizitätsinfrastrukturen; 1,05 Milliarden Euro für $\mathrm{CO}_{2}$-Abscheidungs- und Speicherungstechnologien; 0,565 Milliarden Euro für OffshoreWindenergie) sowie 1,02 Milliarden Euro für Breitbandinternetdienste und neue Herausforderungen (zum Beispiel Klimawandel, erneuerbare Energien, Biodiversität) im ländlichen Raum verwendet werden. 2,6 Milliarden Euro davon kommen aus dem Haushalt 2009, 2,4 Milliarden Euro sollen im Haushalt 2010 bereitgestellt werden. ${ }^{22}$

Die Verhandlungen zur Verwendung dieser vergleichsweise geringen Summe von fünf Milliarden Euro haben einen Vorgeschmack auf die kommenden Haushaltsverhandlungen gegeben. Etliche Mitgliedstaaten haben vor dem Europäischen Rat im Dezember 2008 ihre Zurückhaltung gegenüber einer Öffnung des Mehrjährigen Finanzrahmens geäußert. Insbesondere Deutschland, Finnland, die Niederlande, Italien und Lettland waren gegen eine Änderung des Mehrjährigen Finanzrahmens. Der Europäische Rat vom Dezember 2008 konnte diese Widerstände zunächst nicht auflösen, sodass sich die Mitgliedstaaten in monatelangen Verhandlungen wiederfanden. Die nach dem Europäischen Rat überarbeiteten Vorschläge der Kommission sahen dann auch eine Verringerung des Betrags vor, der durch eine Revision des Mehrjährigen Finanzrahmens zur Verfügung gestellt werden sollte. Außerdem wurde der Bereich Energie und Energietechnologien in den Mittelpunkt der vorzusehenden Maßnahmen gestellt. ${ }^{23}$

Externer Reformdruck hatte somit offenkundig bereits Einfluss auf die Ausrichtung des EU-Haushalts in Richtung zukunftsorientierter Ausgaben. Inwieweit globale Herausforderungen am Ende tatsächlich die Haushaltsverhandlungen beeinflussen werden, bleibt jedoch abzuwarten. Die Wirtschaftskrise hat gezeigt, dass eine Refokussierung des Haushalts möglich ist. Aber selbst, wenn nur ein begrenztes Budget betroffen ist, ist dies mit erheblichem interinstitutionellem Verhandlungsaufwand verbunden. Dies wird sich mit Inkrafttreten des Lissabon-Vertrags nicht ändern und die Mitgliedstaaten werden auch zukünftig nationale Interessen bedienen müssen - unabhängig von der Intensität des externen Reformdrucks.

\section{Mögliche Auswirkungen der Haushaltsüberprüfung auf ausgabenwirksame Gemein- schaftspolitiken}

Die Struktur des EU-Haushalts ist in der mehrjährigen Finanzplanung 2007 bis 2013 wesentlich durch zwei dominante Ausgabenbereiche gekennzeichnet. Dies ist zum einen der Bereich Wettbewerbsfähigkeit und Kohäsion mit einem Ausgabenanteil von 44,2 Prozent sowie zum anderen der Bereich natürliche Ressourcen mit 43 Prozent der Ausgaben. Vor dem Hintergrund dieser Haushaltstitel erschließen sich die derzeitigen Finanzierungsprioritäten somit erst auf den zweiten Blick. Im Haushalt 2009 entfallen im ersten Ausgabenschwerpunkt 48,4 Milliarden Euro der insgesamt knapp 60 Milliarden Euro auf Struktur-

21 Siehe Rat der Europäischen Union: European Economic Recovery Plan - Financing, Dok. 9139/09 (Presse 105).

22 Siehe Agence Europe: EU/Budget: EU27 still disagrees on funding European Economic Recovery Plan, 14.03.2009.

23 Europäische Kommission: Mitteilung der Kommission an das Europäische Parlament, den Rat, den Europäischen Wirtschafts- und Sozialausschuss und den Ausschuss der Regionen. Jetzt investieren in die Zukunft Europas, KOM (2009) 36. 
und Kohäsionsmaßnahmen, aber lediglich 11,1 Milliarden Euro auf Programme zur Förderung der Wettbewerbsfähigkeit (insbesondere das Siebte Forschungsrahmenprogramm, aber auch Investitionen in die Verkehrs- und Energieinfrastruktur). Hinsichtlich der natürlichen Ressourcen werden in 2009 46,7 Milliarden Euro der insgesamt 59,3 Milliarden Euro für Leistungen innerhalb des gemeinsamen Agrarmarktes aufgewendet, die verbleibenden Mittel verteilen sich auf die Entwicklung des ländlichen Raumes, den Umweltschutz und die Fischereipolitik. Somit ist offensichtlich, dass nach wie vor gut 70 Prozent des gesamten EUHaushalts für die Finanzierung der Gemeinsamen Agrarpolitik sowie der Struktur- und Kohäsionsmaßnahmen reserviert sind.

Vor diesem Hintergrund ist leicht erkennbar, dass eine Überprüfung der Finanzierungsprioritäten der Europäischen Union im Sinne der Reallokation von Mitteln für die Stärkung der Wettbewerbsfähigkeit die Gemeinsame Agrar- und die Strukturpolitik als mögliche Umverteilungsquellen und demgegenüber insbesondere die Forschungs- und Technologiepolitik als einen Nutznießer einer neuen Prioritätensetzung betreffen müsste. Deshalb analysieren wir in diesem Abschnitt die bisherigen Ergebnisse der Haushaltsüberprüfung in Bezug auf diese drei Politikfelder. Empirisch stützen wir uns dabei insbesondere auf die Stellungnahmen der mitgliedstaatlichen Regierungen, die im Verlauf der öffentlichen Anhörung in der Zeit von September 2007 bis April 2008 eingegangen sind. Insgesamt wurden im Rahmen dieser Anhörung 94 Stellungnahmen von Regierungsstellen aus 26 Mitgliedstaaten eingereicht. ${ }^{24}$ Der Fokus auf die Darlegungen mitgliedstaatlicher Regierungen begründet sich dabei aus zwei Überlegungen. Erstens erlauben uns diese Stellungnahmen den Raum für einen möglichen Konsens auf Seiten der Mitgliedstaaten einzuschätzen, während zweitens erkennbar wird, welcher strategische Spielraum für die Europäische Kommission bei der Formulierung ihres Vorschlags für die Haushaltsreform besteht.

\section{Gemeinsame Agrarpolitik}

Die Gemeinsame Agrarpolitik der Europäischen Union ist im Prozess der Haushaltsüberprüfung in zweifacher Hinsicht in die Diskussion gekommen: im Hinblick auf die notwendigen Anpassungen der Höhe der Ausgaben sowie in Bezug auf die inhaltliche Ausrichtung dieses Politikfelds.

Anpassung der Ausgaben der Gemeinsamen Agrarpolitik: Zum Verständnis der mitgliedstaatlichen Positionen in diesem Bereich ist darauf hinzuweisen, dass mit der Agrarreform im Rahmen der „Agenda 2000“ im Jahr 1999 die bis dahin dominante Säule der Direktzahlungen an landwirtschaftliche Produktionsbetriebe durch eine zweite Säule der Förderung der Entwicklung des ländlichen Raums ergänzt worden ist. Vor diesem Hintergrund ist im Rahmen der Haushaltsüberprüfung nicht generell eine deutliche Senkung der Ausgaben für die Gemeinsame Agrarpolitik gefordert worden. Vielmehr scheint weithin Konsens darüber zu bestehen, die Direktzahlungen substanziell zu kürzen oder gar auslaufen zu lassen. Die hierdurch freiwerdenden Mittel sollen - und hier divergieren die Auffassungen der Mitgliedstaaten entlang der Konfliktlinie Nettozahler/Nettoempfänger - entweder vornehmlich in die zweite Säule der Gemeinsamen Agrarpolitik oder auch in die Förderbereiche Forschung, Technologie und Innovation umgeleitet werden. Entlang dieser Konfliktlinie finden sich auch Positionen der Mitgliedstaaten, die eine nationale Kofinanzierung der Gemeinsamen

24 Europäisches Parlament: Arbeitsdokument zur Halbzeitüberprüfung des Mehrjährigen Finanzrahmens (20072013) - Aktueller Stand und Perspektiven, PE418.263v01-00 vom 21.01.2009, Berichterstatter: Reimer Böge, S. 4. 
Agrarpolitik für sinnvoll erachten, und solchen, die eine solche mit Verweis auf den , solidarischen' Charakter der Agrarpolitik ablehnen.

Inhaltliche Prioritäten in der Gemeinsamen Agrarpolitik: Aus dem vorher gesagten ergibt sich eine weitgehende Übereinstimmung der mitgliedstaatlichen Positionen zur weitreichenden Reallokation der Mittel der Gemeinsamen Agrarpolitik zugunsten der Förderung der Entwicklung des ländlichen Raums. Insofern dürfte die Haushaltsüberprüfung in diesem Bereich zwei grundlegende Neuausrichtungen befördern. Erstens, eine stärkere Orientierung der Mittel an den Zielen des Klima- und Umweltschutzes sowie der Lebensmittelsicherheit sowie, zweitens, eine durch die Fokussierung auf den ländlichen Raum naheliegende engere konzeptionelle Verzahnung dieses Förderbereichs mit den Fördermaßnahmen im Rahmen der europäischen Regionalpolitik.

\section{Struktur- und Kohäsionspolitik}

In diesem Politikbereich hat die Europäische Kommission, ergänzend zum Konsultationsprozess im Rahmen der Haushaltsüberprüfung, eine inhaltlich stärker spezifizierte Anhörung der mitgliedstaatlichen Positionen durch ihr „Grünbuch zum territorialen Zusammenhalt" vom 6. Oktober 2008 eingeleitet. ${ }^{25}$ Aus diesem Grund beziehen sich zumindest einige der mitgliedstaatlichen Stellungnahmen im Rahmen der Haushaltsüberprüfung bereits auf wesentliche strukturelle Elemente, die erst in dieser Kommissionsmitteilung explizit abgefragt worden sind. Im Kern kann man dadurch aber die Diskussion um die zukünftige Ausgestaltung der Struktur- und Kohäsionspolitik auf folgende zwei Aspekte fokussieren:

Grundlegende Ausrichtung und Finanzierungsprioritäten: Ein in diesem Bereich zu erwartender grundlegender Interessenkonflikt zwischen den wirtschaftlich führenden Staaten und jenen Mitgliedstaaten, die sich hinsichtlich ihrer ökonomischen Leistungsfähigkeit in einem Aufholungsprozess befinden, wird durch die Analyse der mitgliedstaatlichen Stellungnahmen nicht bestätigt. Vielmehr zeigt sich, dass eine Reihe von Staaten der ersten Gruppe (insbesondere Großbritannien und Schweden) ein Auslaufen der Strukturförderung in den reichen Mitgliedstaaten befürworten und damit die Forderungen insbesondere der neuen Mitgliedstaaten nach einer Fokussierung der Mittel auf die Kohäsionsgebiete unterstützen. Demgegenüber befürworten etwa Österreich oder Finnland eine Fortführung der Strukturförderung auch in den wirtschaftlich führenden Mitgliedstaaten soweit sich eine solche insbesondere an den Wettbewerbszielen der Lissabon-Agenda orientiert. ${ }^{26}$

Die Anerkennung, geografisch bedingter Besonderheiten': Dieser Aspekt beleuchtet die Frage, ob spezifische regionalpolitische Maßnahmen als sinnvoll erachtet werden, die sich zugunsten von Bergregionen, Inseln oder dünnbesiedelten Gebieten konzipieren ließen. Erwartungsgemäß werden diesbezügliche Überlegungen von Mitgliedstaaten, die über solche Regionen verfügen (insbesondere Griechenland und Spanien) grundsätzlich begrüßt, während sie im Falle einer, geringeren Betroffenheit' grundsätzlich abgelehnt werden. So verweist etwa die deutsche Bundesregierung in ihrer Stellungnahme darauf, dass die statisti-

25 Europäische Kommission: Mitteilung der Kommission an das Europäische Parlament, den Rat, den Ausschuss der Regionen und den Europäischen Wirtschafts- und Sozialausschuss. Grünbuch zum territorialen Zusammenhalt. Territoriale Vielfalt als Stärke, KOM (2008) 616.

26 Vgl. hierzu: HM Treasury: Global Europe: vision for a 21st century budget, London 2008; Prime Minister's Office Sweden: Reforming the Budget, Changing Europe - Sweden's response to the Commission Communication, Stockholm 2008; Finnish Government: Contribution to the Communication from the Commission "Reforming the budget, changing Europe, a public consultation paper in view of the 2008/2009 budget review", Helsinki 2008; The Austrian Federal Government: EU Budget, Review 2008/2009: Contribution to the Consultation Process, Wien 2008. 
schen Erhebungen der Kommission ergeben hätten, dass solche Gebiete nicht grundsätzlich unter strukturellen Nachteilen litten, zumal sich in allen drei Kategorien sowohl rückständige wie auch wohlhabende Regionen fänden. ${ }^{27}$

\section{Forschungs- und Technologiepolitik}

Unter der Prämisse, dass dieser Politikbereich ein wesentlicher Nutznießer einer Reallokation von Mitteln sein dürfte, erscheinen politikfeldspezifische Implikationen der Haushaltsüberprüfung in folgenden Dimensionen möglich:

Forschungsfelder: In diesem Bereich belegt die öffentliche Anhörung einen zunehmenden Konsens in Richtung einer Fokussierung europäischer Forschungsförderung auf eine relativ geringe Zahl strategischer Felder. Zu diesen Feldern gehören zweifellos neue Energieund Umwelttechnologien. Offen erscheint aber noch, ob ein solcher Fokus das Portfolio des Siebten Forschungsrahmenprogramms ergänzen soll oder ob tatsächlich eine Beschränkung europäischer Forschungsförderung auf wenige strategische Bereiche durchsetzbar ist. Eine solche Fokussierung könnte sich immerhin auf einen Bericht einer Expertengruppe der Europäischen Kommission stützen, die sich für eine Bündelung der Mittel auf die Forschungsbereiche E-Health, pharmazeutische Forschung, Energie und digitale Inhalte ausgesprochen hat. ${ }^{28}$

Finanzierung von Forschung und Entwicklung: Im Grundsatz fordern sowohl die Mehrheit der Mitgliedstaaten, die Europäische Kommission und das Europäische Parlament die Erhöhung der Mittel für Forschung und Entwicklung. Im Oktober 2009 hat die Kommission im Rahmen des sogenannten „SET-Plans“ beispielsweise vorgeschlagen, im kommenden Jahrzehnt aus Mitteln öffentlicher und privater Akteure nicht weniger als 50 Milliarden Euro in die Entwicklung umweltschonender Technologien zu investieren. Damit könnten nach Ansicht der Kommission allein in den Sektoren der Wind-, Solar- und Bioenergietechnologien immerhin mehr als 400.000 Arbeitsplätze geschaffen werden. ${ }^{29}$

Der Forderung nach mehr Forschungsmitteln steht entgegen, dass insbesondere einige mitgliedstaatliche Regierungen in gleicher Weise die Auffassung vertreten, die europäische Forschungsförderung müsse sich stärker auf die Generierung eines europäischen Mehrwerts konzentrieren und dabei insbesondere dem Subsidiaritätsprinzip folgen. Hieraus wird ersichtlich, dass die Diskussion um höhere Investitionen in einem unmittelbaren Zusammenhang mit den zukünftigen grundlegenden Zielen der europäischen Forschungspolitik stehen wird.

Innerhalb der aktuellen Struktur des Siebten Forschungsrahmenprogramms lassen sich zudem Intentionen einzelner Mitgliedstaaten identifizieren, einen größeren Teil der Ausgaben für den Programmbereich Ideen und damit dem Europäischen Forschungsrat zur Verfü-

27 Gemeinsame Stellungnahme des Bundes und der Länder zur Mitteilung der Europäischen Kommission „Grünbuch zum territorialen Zusammenhalt - Territoriale Vielfalt als Stärke“, Berlin 2009, S. 4.

28 Ein innovatives Europa schaffen. Bericht der unabhängigen Sachverständigengruppe für F\&E und Innovation, eingesetzt im Anschluss an das Gipfeltreffen in Hampton Court, Luxemburg 2006. Ein anderes Beratungsgremium der Kommission, das European Area Research Board, schlägt vor, die Anstrengungen innerhalb des Europäischen Forschungsraums auf die Bereiche des Klimawandels, der Energie- und Wasserversorgung, der Problematik der alternden Gesellschaften, der Gesundheitsvorsorge und der nachhaltigen Wohlstandssicherung zu konzentrieren. Europäische Kommission: Preparing Europe for a New Renaissance. A Strategic View of the European Research Area. First Report of the European Research Area Board, Brüssel 2009.

29 Europäische Kommission: Mitteilung der Kommission an das Europäische Parlament, den Rat, den Europäischen Wirtschafts- und Sozialausschuss und den Ausschuss der Regionen. Investitionen in die Entwicklung von Technologien mit geringen $\mathrm{CO}_{2}$-Emissionen (SET-Plan), KOM (2009) 519. 
gung zu stellen. ${ }^{30}$ Um dadurch das Investitionsniveau in den anderen Programmbereichen nicht absenken zu müssen, könnten jene Förderbereiche vor allem durch Zuflüsse aus anderen Haushaltstiteln oder aus Förderaktionen innerhalb des Siebten Forschungsrahmenprogramms, die sich bisher als wenig effektiv erwiesen haben, kompensiert werden. ${ }^{31}$

Schließlich existiert im Grundsatz weitgehende Einigkeit über die Notwendigkeit, aus bisherigen Mitteln der Gemeinsamen Agrarpolitik neue Vorhaben in den Bereichen Forschung, Innovation und Energie zu finanzieren. ${ }^{32}$ Dies lassen insbesondere die Stellungnahmen der Mehrheit der mitgliedstaatlichen Regierungen erkennen, ohne dass daraus schon ersichtlich würde, in welchem Umfang eine solche Reallokation von Mitteln aus anderen Haushaltstiteln erreicht werden kann. In diesem Zusammenhang ist aber wichtig zu sehen, dass bereits parallel zur Haushaltsüberprüfung Mittel innerhalb größerer Ausgabenbereiche in diese Richtung umdelegiert werden, ohne sie formal dem Bereich Wettbewerbsfähigkeit zuzuordnen. So hat etwa die Europäische Kommission in einer Mitteilung zur Strukturpolitik ihre Erwartung ausgedrückt, dass zukünftig wesentliche Mittel dieses Bereiches dem Ziel der Forschungs- und Innovationsförderung entsprechen sollen. ${ }^{33}$

Grundlegende Ziele der Forschungs- und Technologiepolitik: Der Konsultationsprozess zur Haushaltsüberprüfung hat deutlich gemacht, dass dem Ziel der Generierung eines europäischen Mehrwerts in der Forschungs- und Technologiepolitik in Zukunft ein größerer Stellenwert eingeräumt werden dürfte. ${ }^{34}$ Dabei sehen insbesondere die mitgliedstaatlichen Regierungen einen solchen Mehrwert in der Förderung exzellenter Forschung, der Stärkung transnationaler Forschungsnetzwerke und der Förderung von Programmen für lebenslanges Lernen. Insofern decken sich diese Mehrwertzuschreibungen im Wesentlichen mit den bisherigen Zielen der europäischen F\&E-Politik, sodass die ,Mehrwert-Diskussion` kaum zu einer Reformulierung dieser Ziele führen wird, wohl aber Auswirkungen auf die Ausgestaltung auf programmlicher und instrumenteller Ebene haben kann.

\section{Reformperspektiven für eine zukunftsfähige Ausgabenstruktur: drei Szenarien}

Vorangehend haben wir argumentiert, dass die EU-Haushaltsüberprüfung zu einer Vorfestlegung von Verhandlungspositionen der im Jahr 2011 anvisierten Haushaltsreform führen kann. Dies würde bedeuten, dass die Haushaltsüberprüfung zwar nicht unmittelbar eine Änderung der Ausgabenstruktur bewirken würde, dass sie zukünftige Schwerpunkte (sei es thematisch oder bezogen auf die Instrumente) jedoch präjudizieren könnte. Allerdings ist zu bedenken, dass interner Reformbedarf und externer Reformdruck wichtige Einflussfaktoren für einen möglichen Wandel der Ausgabenstruktur sind. Momentan ist die Diskrepanz zwischen Rhetorik und Realität jedoch noch groß: zwar sieht ein Großteil der EU-Mitglieder die Ziele der Lissabon-Strategie, insbesondere im Bereich Forschung und Entwicklung, als wichtigste Herausforderung an, tatsächlich ist jedoch weiterhin kein Fortschritt bei der Er-

30 Ministry of Foreign Affairs of the Netherlands: Dutch priorities for a modern EU budget, Den Haag 2008, S. 5.

31 Torbjörn Danell/Anders Östhol: The EU Long-term Budget. Reform and New Priorities, Swedish Institute for Growth Policy Studies 2008:002.

32 Europäisches Parlament: Arbeitsdokument, 2009, S. 5.

33 Europäische Kommission: Mitteilung der Kommission an das Europäische Parlament, den Rat, den Europäischen Wirtschafts- und Sozialausschuss und den Ausschuss der Regionen. Kohäsionspolitik: In die Realwirtschaft investieren, KOM (2008) 876/3, S. 9.

34 Siehe hierzu Robert Kaiser: Mehrwert und Gemeinwohl in der europäischen Forschungs- und Technologiepolitik, in: Gerold Ambrosius/Peter Schmitt-Egner (Hrsg.): Europäisches Gemeinwohl - Historische Dimension und aktuelle Bedeutung, Baden-Baden 2006, S. 167-182. 
reichung der Ziele, insbesondere des ,Drei-Prozent-Ziels“ für Investitionen in Forschung und Entwicklung, erkennbar. ${ }^{35}$

Eine Reform des EU-Haushalts in Richtung einer zukunftsorientierten, das heißt einer auf die (Post-)Lissabon-Agenda und globale Herausforderungen ausgerichteten Ausgabenstruktur, ist von einer Reihe externer, aber vor allem auch interner nationaler Faktoren abhängig, die teilweise bereits erläutert wurden. Die Frage, die sich daran anschließt, ist, unter welchen Konstellationen oder Bedingungen Blockaden auflösbar sind, sodass eine eher zukunftsorientierte Ausgabenstruktur realisiert werden kann. Diese Reformperspektiven, die unterschiedliche Ausmaße des Wandels in Bezug auf Strukturen, Prozesse und Akteure widerspiegeln, werden im Folgenden in Form von drei Szenarien beschrieben.

\section{Szenario 1: ,Normale Anpassung “ - Politikwandel erster Ordnung}

Dieses Szenario zeichnet sich durch die Stabilität des vorherrschenden Politikparadigmas aus. Eine Anpassung des Haushalts, seiner Struktur und Instrumente aufgrund interner und externer Herausforderungen erfolgt allenfalls inkrementell. In diesem Szenario würden die Mitgliedstaaten weitgehend ,konservativ` agieren, das heißt gewohnte Reaktionsmuster zeigen und alte Privilegien verteidigen, während die Kommission einzig darauf bedacht wäre, Konflikte zwischen Mitgliedstaaten und mit anderen EU-Institutionen zu begrenzen. Als Resultat würden zukunftsorientierte, das heißt auf Forschung, Technologie und Innovation ausgerichtete Programme, wie zum Beispiel das Siebte Forschungsrahmenprogramm, hinsichtlich ihrer Struktur, Inhalte, Instrumente und Finanzierung weitgehend unverändert bleiben. Allenfalls eine stärkere Nutzung bestimmter bislang vernachlässigter Instrumente, wie der Europäischen Investitionsbank, sowie im F\&E-Bereich eine Fokussierung auf Themen von globaler Dimension ist zu erwarten. Sollte es keine signifikante Reallokation von Finanzmitteln zwischen Politikbereichen geben, wäre es in diesem Szenario dennoch denkbar, dass bestimmte Politiken, insbesondere die Gemeinsame Agrarpolitik sowie die Strukturpolitik, stärker mit der Lissabon-Strategie in Einklang gebracht werden könnten.

\section{Szenario 2: Intra-sektorale Anpassung - Politikwandel zweiter Ordnung}

Die zentrale Voraussetzung für dieses zweite Szenario wäre, dass die Kommission sowohl mit den Ergebnissen der Haushaltsüberprüfung, inklusive der Konsultation, als auch den Erfordernissen globaler Herausforderungen argumentiert, um ambitiösere Vorschläge für einen zukünftigen EU-Haushalt vorzulegen. In dieser Reformperspektive würde es der Kommission als zentralem Agenda-Setter gelingen, für signifikante Änderungen in der Ausgabenstruktur innerhalb der einzelnen Politikbereiche (Budgetlinien) zu argumentieren. ${ }^{36}$ Eine derartige Reform könnte zum Beispiel im Bereich Forschung und Innovation zu einer Fokussierung auf Themen führen, in welchen eine kritische Masse zu deren Bearbeitung nur

35 Dies bezieht sich allerdings auf einen EU-27-Vergleich der Jahre 2000 und 2006, das heißt ein wichtiger Erklärungsfaktor für die Stagnation ist die Erweiterung der Europäischen Union im Jahre 2004 um Staaten mit schwacher F\&E-Intensität. Allerdings stagnierte auch die F\&E-Intensität in den vier großen Mitgliedern Deutschland, Vereinigtes Königreich, Frankreich und Italien. In 17 EU-Mitgliedstaaten stieg hingegen die F\&E-Intensität seit dem Jahr 2000. Siehe Europäische Kommission: Companion document. Implementation of the Lisbon Strategy Structural Reforms in the context of the European Economic Recovery Plan - a more detailed overview of progress across the EU in the specific macro- and micro-economic as well as the employment areas, COM (2009) 34/2, Volume II, S. 27-29.

36 Das European Research Area Board geht davon aus, dass die unmittelbaren Ausgaben für Forschung und Entwicklung am Gesamthaushalt im Jahr 2030 zwölf Prozent ausmachen werden, während knapp ein Drittel der Strukturfondsmittel ausschließlich für F\&E vorgehalten werden sollen. Siehe Europäische Kommission: Preparing Europe for a New Renaissance, 2009. 
auf europäischer Ebene erreicht werden kann. Eine angemessene Finanzierung müsste durch eine flexiblere Budgetstruktur garantiert werden, welche es ermöglicht, zusätzliche Finanzquellen zum Beispiel durch öffentlich-private Partnerschaften oder die Europäische Investitionsbank zu erschließen. Etliche dieser Reformen könnten bereits innerhalb der bestehenden Programme umgesetzt werden.

Mittelfristig ist es auch denkbar, dass etablierte Programmstrukturen, wie zum Beispiel die des Forschungsrahmenprogramms, aufgelöst und administrative Aufgaben der Kommission in Agenturen ausgelagert werden. Diese Strategie könnte jedoch bei EU-Mitgliedern, die von den derzeitigen Programmstrukturen stark profitieren und die befürchten müssten, dass eine mehr auf die Lissabon-Strategie ausgerichtete Ausgabenstruktur zu ihrem Nachteil wäre, auf Widerstand stoßen. Als Ausweg könnte die Kommission solche Ausgleichsmaßnahmen vorschlagen, die auf die Stärkung von Forschungs- und Innovationskapazitäten sowie Forschungs- und Innovationsinfrastrukturen zielen. In der Gemeinsamen Agrar- und der Strukturpolitik würde dieses Szenario im Wesentlichen auf eine klare Fokussierung der Mittel auf die Förderung des ländlichen Raums und der Kohäsionsgebiete hinauslaufen.

\section{Szenario 3: Ein neues Politikparadigma - Politikwandel dritter Ordnung}

Die Etablierung eines neuen Politikparadigmas würde zunächst voraussetzen, dass eine bestimmte Anzahl von Mitgliedstaaten eine Führungsrolle im Hinblick auf eine reformierte Ausgabenstruktur des EU-Haushalts übernimmt, um diesen deutlich auf die LissabonAgenda auszurichten. Ein derartiges Szenario würde ebenso eine bemerkenswerte Umverteilung zwischen den Budgetlinien erfordern, wie auch einen deutlichen Schwerpunkt der Ausgaben auf eine ausgesuchte Anzahl von Politiken, in welchen ein europäischer Mehrwert unbestritten ist.

Wahrscheinlich ist, dass eine derartige Reform den Widerstand sowohl jener Mitgliedstaaten hervorrufen würde, welche nicht zur Führungsgruppe gehören, als auch einiger Generaldirektorate der Europäischen Kommission, da ein Paradigmenwechsel notwendigerweise ,Haushaltsgewinner" und ,Haushaltsverlierer" erzeugen würde.

Folglich ergeben sich aus diesem Szenario drei entscheidende Fragen: a) Welche Mitgliedstaaten wären Anwälte eines derartigen Paradigmenwechsels? b) Welche Kompensationsmechanismen stünden für diejenigen Mitgliedstaaten zur Verfügung, die diesen Wandel nicht unterstützen? c) Warum sollte die Europäische Kommission dem Paradigmenwechsel, angesichts möglicher interner Widerstände, zustimmen?

Ebenso wahrscheinlich ist, dass in jedem Fall jene Mitgliedstaaten einen Wandel in Richtung zukunfts- und innovationsorientierter Ausgaben, das heißt vor allem von Agrar- und Strukturausgaben hin zu Forschungs- und Innovationsinvestitionen, vorantreiben würden, welche bereits starke Forschungs- und Innovationskapazitäten aufweisen können. Wie in einigen Beiträgen zum Konsultationsprozess bereits angedeutet, könnten andere Mitgliedstaaten dem zustimmen, sollten zum Beispiel Nettozahler zum EU-Haushalt bereit sein, die Obergrenze des EU-Haushalts flexibler zu handhaben. Diese zusätzlichen Finanzmittel könnten an Staaten mit schwächeren Forschungs- und Innovationssystemen für die Kapazitätsbildung zurückfließen.

Die tatsächliche Zustimmung der Kommission zu einem Paradigmenwechsel ist eng mit dem Machtspiel zwischen dieser, dem Europäischen Parlament und den Mitgliedstaaten beziehungsweise der EU-Präsidentschaft verbunden. Die zweite Hälfte des Jahres 2009 hat diesbezüglich ein potenzielles ,Politikfenster' für einen Wandel geöffnet.

Zum Ersten ist die Europäische Kommission in eine Art ,Ruhephase ' eingetreten, in welcher die amtierenden Kommissare lediglich die Geschäfte verwaltet haben, neue Entschei- 
dungen jedoch nicht getroffen wurden. Zweitens fing das im Juni 2009 neu gewählte Europäische Parlament erst im Herbst 2009 an inhaltlich zu arbeiten. Und drittens schließlich hatte Schweden die EU-Präsidentschaft in der zweiten Hälfte 2009 inne. Schweden ist ein ausgesprochener Verfechter einer Reform des EU-Haushalts in Richtung der LissabonAgenda und hat die sechs Monate genutzt, um eine Reform in diese Richtung voranzutreiben.

Die beschriebene Konstellation hat einen Paradigmenwechsel zumindest näher gebracht. Unter schwedischer Präsidentschaft wurden die Prioritäten der Europäischen Union deutlich in Richtung eines zukunftsorientierten Haushalts formuliert, indem unter anderem ein Politikbereiche übergreifender Ansatz gefordert wurde, der insbesondere Antworten auf die groBen globalen und sozialen Herausforderungen finden soll. ${ }^{37}$

\section{Fazit: Politikwandel jenseits des Inkrementalismus}

Eine abschließende Bewertung der Realisierungsoptionen für die genannten drei Szenarien deutet derzeit auf einen Politikwandel zweiter Ordnung hin und damit auf die Fähigkeit der Europäischen Union, den beschriebenen inneren und äußeren Herausforderungen zumindest durch eine Veränderung ihrer Haushaltsstruktur jenseits inkrementeller Anpassung gerecht zu werden. Vieles spricht dafür, dass die bisher dominanten Ausgabenbereiche der Europäischen Union mittelfristig erheblichen Veränderungen unterworfen sein dürften. So werden in der Gemeinsamen Agrar- und der Strukturpolitik nicht nur etablierte Subventionslogiken infrage gestellt, insbesondere werden vor allem auch mit der Ausrichtung auf die Lissabon-Agenda und/oder die neuen globalen Herausforderungen Wege für eine Neuausrichtung der Finanzstruktur innerhalb dieser Politikbereiche, aber auch im Verhältnis zwischen verschiedenen Ausgabenbereichen aufgezeigt. Eine intra-sektorale Anpassung wird in dem Maße zunehmend wahrscheinlich, in dem die Mehrheit der Mitgliedstaaten, aber auch die Europäische Kommission und das Europäische Parlament die Notwendigkeit steigender Ausgaben für Forschung, Entwicklung und Innovation akzeptieren; sie gleichzeitig aber auch durch die Infragestellung von Direktzahlungen in der Gemeinsamen Agrarpolitik sowie der Strukturförderung in wirtschaftlich führenden Mitgliedstaaten eine nicht unerhebliche Umverteilungsmasse definieren.

Dies alles ist jedoch von einem grundlegenden Paradigmenwechsel weit entfernt. Zweifellos gewinnen in der Europäischen Union ambitionierte Reformstrategien wieder an Bedeutung. Aufgrund ihrer langen zeitlichen Perspektiven üben sie aber weder auf die Gemeinschaftsorgane noch die Mitgliedstaaten unmittelbaren Veränderungsdruck aus. Offenkundig ist aber auch, dass die Europäische Union aufgrund ihrer komplexen und konsensorientierten Verhandlungs- und Entscheidungsstruktur grundsätzlich ungünstige Bedingungen für Politikanpassungen in kurzer zeitlicher Perspektive bietet. Dies spricht nicht gegen die Annahme, dass im Jahr 2009 ein ,Politikfenster' für grundlegende Veränderungen existiert hat. Allerdings ist unverkennbar, dass der strategischen Nutzung eines solchen durch die Parallelität nationaler und europäischer Politiklogiken von vornherein enge Grenzen gesetzt sind.

37 Rat der Europäischen Union: Conclusions on guidance on future priorities for European research and researchbased innovation. 2982nd Competitiveness Council meeting. Brussels, 3 December 2009. 